\title{
Functional Rather than Effector-Specific Organization of Human Posterior Parietal Cortex
}

\author{
Tobias Heed, ${ }^{1 \star}$ Sabine M. Beurze, ${ }^{2}$ Ivan Toni, ${ }^{2}$ Brigitte Röder, ${ }^{1}$ and W. Pieter Medendorp ${ }^{2}$ \\ ${ }^{1}$ Biological Psychology and Neuropsychology, Department of Psychology, University of Hamburg, 20146 Hamburg, Germany, and ${ }^{2}$ Radboud University \\ Nijmegen, Donders Institute for Brain, Cognition and Behaviour, 6525 HR Nijmegen, The Netherlands
}

\begin{abstract}
Neurophysiological and neuroimaging studies have shown that the posterior parietal cortex (PPC) distinguishes between the planning of eye and hand movements. This distinction has usually been interpreted as evidence for a modular, effector-specific organization of this cerebral region. However, the eyes differ markedly from other body parts both in terms of their functional purpose and with regard to the spatial transformations required to plan goal-directed movements. PPC may therefore provide specialized subregions for eye movements, but distinguish less for other effectors. Using functional magnetic resonance imaging, we compared activity during memoryguided eye, hand, and foot movements in human participants. The results did not reveal any significant activation differences during the planning of hand and foot movements, except in the most anterior part of PPC [Brodmann's area (BA) 5], marginally extending into anterior BA 7/40. This region showed a lateral-to-medial gradient for hand versus foot movement planning. The limb-unspecific PPC regions were functionally connected with hand and foot motor regions. In contrast, a gradient-like organization was found for all of PPC for the planning of eye versus hand and foot movements. Although planning-related activity across the three effectors considerably overlapped, saccade planning activated occipitoparietal regions more than limb movements, whereas limb movements activated anterior regions of the superior parietal lobule more than saccades. We infer that PPC does not follow a strict effector-specific organization. Rather, the large-scale organization of this region might reflect the different computational constraints that need to be satisfied when planning eye and limb movements.
\end{abstract}

\section{Introduction}

The posterior parietal cortex (PPC) is important for coordinate transformations for spatially guided motor behavior. In both humans and nonhuman primates, different portions of the PPC are preferentially active for preparing different types of actions or for movements involving different body parts (Hyvärinen, 1981; Murray and Coulter, 1981; Graziano and Gross, 1998; Andersen and Cui, 2009; Caminiti et al., 2010). In the intraparietal sulcus (IPS) of macaques, neurons in the lateral intraparietal area (LIP) respond preferably during the planning of saccades, whereas neurons in the adjacent parietal reach region (PRR) respond more strongly during arm reach planning (Calton et al., 2002; Cui and Andersen, 2007; Andersen and Cui, 2009). Neither region, however, appears to respond exclusively to either effector (Snyder et al., 1997; Andersen and Cui, 2009). Accordingly, research using functional magnetic resonance imaging (fMRI) in humans has revealed a large overlap of PPC activation during the planning of

Received Aug. 20, 2010; revised Dec. 2, 2010; accepted Dec. 27, 2010.

W.P.M. is funded by the Human Frontier Science Program and the Netherlands Organization for Scientific Research. We thank Pascal de Water and Jos Wittebrood for technical assistance with the infrared light-emitting diode setup, and Paul Gaalman for expert assistance with magnetic resonance imaging recording. We thank Lennart Verhagen for providing Matlab code for the extraction of CSF values from magnetic resonance data.

${ }^{*}$ T.H. has previously published under the name Tobias Schicke.

Correspondence should be addressed to Tobias Heed, Biological Psychology and Neuropsychology, Department of Psychology, University of Hamburg, von-Melle-Park 11, 20146 Hamburg, Germany. E-mail: tobias.heed@uni-hamburg.de.

DOI:10.1523/JNEUROSCI.4370-10.2011

Copyright (C) 2011 the authors $\quad 0270-6474 / 11 / 313066-11 \$ 15.00 / 0$ eye and hand movements (Beurze et al., 2007, 2009; Levy et al., 2007; Hinkley et al., 2009). However, biases for either eye or arm movements have also been shown in humans (Connolly et al., 2003; Medendorp et al., 2005; Connolly et al., 2007; Hinkley et al., 2009; Van Der Werf et al., 2010), and candidate homologs for macaque areas LIP and PRR have been proposed (Connolly et al., 2003; Schluppeck et al., 2005; Beurze et al., 2007; Hagler et al., 2007). These findings have led to the view that, analogously to the somatotopy of primary somatosensory cortex (Penfield and Boldrey, 1937), PPC is organized in an effector-specific manner, with distinct subregions mediating spatially guided movements for each effector (Andersen and Cui, 2009; Hinkley et al., 2009).

However, this conclusion about PPC organization may be premature (Hagler et al., 2007; Levy et al., 2007). Functionally, eye movements are inseparably bound to visual perception and serve a different purpose than movements of any other body part; parietal regions might be differently sensitive to these functions. For example, the sensory reference frame imposed by the retina for visual target processing is linked to the motor reference frame of the eyes. In contrast, the reference frame of a visual target is distinct from the motor reference frame of a limb (Graziano et al., 1997; Ferraina et al., 2009). Therefore, specifying a goal-directed limb movement requires additional transformations to compute the spatial relationship between target and effector position, which depends on the current body posture (Buneo et al., 2002; Beurze et al., 2006). Although effector-specific organization of PPC has been questioned on the basis of overlapping activity of eye- and hand-related activity (Hagler et al., 2007; Levy et al., 
2007), experiments involving only two effectors do not allow deciding between effector specificity versus other, functionally based explanations as the reason of existing regional activity biases in PPC.

Here we used AMRI to compare PPC responses while participants planned goal-directed movements with their eyes, their right hand, or their right foot. We tested whether each effector recruits specific subregions of PPC, or whether only saccades, compared with any limb movements, are specially processed.

\section{Materials and Methods}

Participants

The dataset comprised 23 healthy, right-handed and right-footed participants ( 12 female), aged 19-39 (mean 23.5) years old, with normal or corrected-to-normal vision. The experiment was conducted according to the guidelines of the Declaration of Helsinki and to the institutional guidelines of the local ethics committee (Committee on Research Involving Human Subjects, region Arnhem-Nijmegen, The Netherlands).

\section{Eye tracking}

Position of the left eye was recorded using a long-range infrared videobased eyetracker (SensoMotoric Instruments) at a frequency of $50 \mathrm{~Hz}$. Saccades were identified by detecting a $2 \%$ change from baseline of eye position in the horizontal direction relative to the maximum amplitude of the trial and were verified visually. The reaction time (RT) of correct saccades was calculated as the time between movement cue and saccade onset. Saccade amplitude was measured as the distance traveled by the eye between the average of the four sampling points immediately preceding the movement cue and the eye movement's endpoint. Saccade amplitudes were used to correlate target eccentricity with eye movement amplitude within each participant to ascertain that participants made accurate eye movements and did not just indicate whether the target had been left or right. Fisher $Z$-transformation was used in all statistical tests involving correlations. Eye tracker data were not available for two participants. All reports on eye movement performance include the 21 remaining participants.

\section{Hand and foot movement recording}

Infrared light-emitting diodes (LEDs) were taped to the right index finger and to the big toe of the right foot. Their light was visible to a video camera, but not to the participants. The video camera was positioned at a distance of about $2 \mathrm{~m}$ from the scanner. Short interruptions of the LEDs, synchronized with the events of the trials, were used for synchronization of the video recording with the eye movement data and the experiment's events. LED locations were extracted from each video frame using MATLAB, rendering a movement time course for both hand and foot movements. Movements were identified by detecting a $5 \%$ change from baseline of hand or foot position relative to the maximum amplitude of the current trial, and were verified visually.

Analysis of these time courses was approached similarly as the analysis of eye movements. Reaction time for correct movements was calculated as the time between movement cue onset and movement onset. Movement amplitude of correct movements was extracted as the distance (in pixels in the image) between limb location before the movement cue and the movement endpoint. Movement amplitude for hand and foot was scaled arbitrarily (depending on the distance and zoom of the camera). Performance was therefore assessed by correlating target eccentricity and movement amplitude.

\section{MRI measurement}

Functional MRI measurement. Functional images were acquired on a Siemens 3 tesla MRI system (Tim TRIO, Siemens). Using an eightchannel phased array head coil, 26 axial slices were obtained by a gradient echo-planar imaging sequence [slice thickness $3 \mathrm{~mm}$, gap $=17 \%$, inplane pixel size $3 \times 3 \mathrm{~mm}$, repetition time $(\mathrm{TR})=1780 \mathrm{~ms}$, echo time $(\mathrm{TE})=30 \mathrm{~ms}$, field of view $(\mathrm{FOV})=224 \mathrm{~mm}$, flip angle $=90^{\circ}$, covering all of the parietal lobe, the motor-related areas of the frontal lobe, and the majority of the occipital lobe.
Anatomical MRI measurement. After the functional recordings, highresolution anatomical images were acquired using a T1-weighted MPRAGE (magnetization prepared rapid gradient echo) sequence (176 sagittal slices, voxel size $=1 \times 1 \times 1 \mathrm{~mm}, \mathrm{TR}=2300 \mathrm{~ms}, \mathrm{TE}=3.93 \mathrm{~ms}$, $\mathrm{FOV}=256 \mathrm{~mm}$, flip angle $=8^{\circ}$ ).

\section{Experimental setup and task design}

Participants lay supine in the scanner. Their head was stabilized with foam cushions. The right arm was cushioned for stabilization. The right hand was placed near the right hip bone, on the right side of the body. The index finger was extended, and pointing was executed by moving both the index finger and the wrist joint, but not the rest of the arm. The right leg was cushioned, and leg and hip were strapped to minimize hip movement during foot pointing. For foot pointing, participants pointed with the big toe by moving the ankle. Participants had to fixate a central white circle throughout the experiment. The right hand and foot had to point toward the fixation point only in the horizontal direction (i.e., left-right), but not in the vertical direction, because vertical pointing movements were very uncomfortable to participants.

Visual targets were projected via a mirror so that they appeared to be located approximately above the participants' head. Targets consisted of small, light gray, filled circles. Pointing movements were made to the apparent location of the target and without seeing the limbs, so that target processing and general task requirements were identical for all conditions. We used a multiple-stage delayed-instruction paradigm (Hoshi and Tanji, 2000; Beurze et al., 2007, 2009) to separate eye, hand, and foot movements. A normal trial consisted of the following four phases: a fixation phase, an effector-planning phase, a target-planning phase, and a movement phase. In $25 \%$ of the trials, the movement phase was omitted (i.e., no-go trials) to reduce the correlation of planning and execution predictors in the general linear model (GLM) (see Preprocessing and statistical analysis section). Importantly, the order of the two planning phases could be either effector-target or target-effector, allowing a dissociation of these two trial parts in our analyses. The experimental design is illustrated in Figure 1.

Each trial began with a fixation phase $(2-4 \mathrm{~s})$, indicated by a short blinking of the fixation point. The effector for each trial was instructed by a color change of the fixation point and a small letter $(\mathrm{O}, \mathrm{H}$, or $\mathrm{V}$ for the first letter of the Dutch words for eye, hand, and foot) projected into the fixation point; both were presented for $350 \mathrm{~ms}$. The start of the next trial phase was either $2-5 \mathrm{~s}$ (when the effector was cued first) or 2-6 s (when the effector was cued second) after the start of the effector phase.

The target was presented for $350 \mathrm{~ms}$ at the same vertical location as the fixation point, but horizontally displaced to the left or right by a 4.4, 5.4, 6.4 , or $7.4^{\circ}$ visual angle. It was masked by a large number of identical circles (presentation time $66 \mathrm{~ms}$ ). Participants had to keep the exact location of the target in memory and prepare an accurate movement of the cued effector. The start of the next trial phase was either $2-5 \mathrm{~s}$ (when the target was presented first) or 2-6 s (when the target was presented second) after the start of the target phase.

Movement execution was cued by the fixation point turning purple for $350 \mathrm{~ms}$. Participants were instructed to move the cued effector as soon as the movement cue occurred, and to make small, reasonably fast movements to the horizontal target location and back to the resting position. The next trial started 2-4s after the movement cue. In no-go trials, the fixation point never turned purple but just blinked to indicate the start of a new trial. For these trials, participants were instructed to simply start over with the upcoming trial. There was a 20 s pause after every 20 trials, during which subjects could freely move their eyes.

The experiment consisted of 192 trials, divided into 64 trials for each effector. Of those 64 trials, 32 had leftward targets, and the other 32 had rightward targets. Each target eccentricity was equiprobable. Of the 64 trials, 16 were no-go trials ( 8 leftward, 8 rightward). Participants practiced the task before going into the scanner until they had clearly understood the task, the meaning of the cues, and had learned to follow the fixation instructions. The experiment was controlled with Presentation, version 12 (Neurobehavioral Systems). 


\section{Trial selection}

Only correctly executed trials were used in the behavioral and fMRI analyses. To this end, kinematics of eye, hand, and foot movements were inspected visually for each trial. Trials in which movement was directed to the wrong side were discarded. Similarly, trials in which a wrong effector had been moved (e.g., a saccade was made together with the pointing, or the hand was moved instead of the foot) were discarded. Finally, trials were removed from further analyses when movement occurred before the movement cue.

\section{fMRI data analysis}

Preprocessing and statistical analysis. Data were analyzed with BrainVoyager QX, versions 1.10.4 and 2.0 (Brain Innovation), and Matlab (MathWorks). Data were preprocessed to correct for slice scan time, head motion, and low-frequency artifactual drift. The first three images of each functional run were discarded to allow for saturation effects at the beginning of scanning.

Functional images were coregistered with the anatomical scan and transformed into Talairach coordinate space. Each hemisphere was then segmented at the gray/white matter boundary, and the cortical sheet of each hemisphere was reconstructed, inflated, and morphed into a sphere (Goebel et al., 2006). The same-side hemispheres of all participants were then aligned based on their cortical folding pattern (van Atteveldt et al., 2004). Then, rather than analyzing spatially identical voxels in all participants, statistical analyses were run over corresponding vertices of the reconstructed hemispheres, based on the cortex-based alignment. This method improves statistical power by increasing the cortical overlap between individual brains (Fischl et al., 1999; Goebel et al., 2006). It also allows analysis without smoothing of the functional data.

Data were analyzed using a standard GLM. A random-effects group analysis was performed to test the effects across subjects. Multiple comparisons were controlled for by the false discovery rate (FDR) procedure with a threshold value of 0.05 , unless noted otherwise. Thirty-six boxcar predictor functions were modeled to capture the different events in the experiment and convolved with a gamma function that modeled the hemodynamic response of the blood oxygenation level-dependent (BOLD) signal. The fixation phase of each trial served as baseline. The fixation phases following no-go trials were modeled with a separate predictor to ensure that activity related to the undoing of a motor plan (Brown et al., 2008) would not contaminate the baseline. For the period of the first cue, there were five predictors (first cue was a left target or a right target; first cue indicated a hand, foot, or eye movement). For the period of the second cue, there were 12 predictors, one for each combination of effector (eye, hand, or foot), target (left or right), and cue order (effector first, target first). For the movement phase, there were three predictors, one for each effector; intervals for the movement predictors were based on the timing of the movement cue, rather than on actual movement. There were predictors for incorrect trial phases (fixation, cue 1 effector, cue 1 target, cue 2, movement), which coded for all trial phases that could not be used for analysis (e.g., because of erroneous movements). Separate predictors were used for the period before the first trial started at the beginning of a run and for the 20 s pauses. To account for head movement during the course of the experiment, $z$-standardized and low-pass-filtered time courses of the movement correction were used (3 predictors for rotational movements, and 3 predictors for translational movements). Head motion was always $<3 \mathrm{~mm}$. Finally, the average signal of all voxels that covered the ventricles was extracted for each time point to form a predictor for the CSF; this predictor allowed correction for signal changes due to mass dislocation of the arm and foot during pointing (Diedrichsen et al., 2005).

Saccades always involve both eyes, whereas pointing in this study involved only the right hand and foot, possibly leading to stronger lateralization of activation for hand and foot than for eyes. We therefore did not analyze hemispheric biases of any condition. More importantly, a hemispheric bias for limb movements does not affect posterior-to-anterior differences between conditions reported here. This holds also for any lateralization differences that may arise from the limbs being positioned on the right side of the body during the pointing task, as in some previous studies (Medendorp et al., 2005; Hagler et al., 2007).
Gradient maps. The difference between the $\beta$ weights of conditions of interest was visualized by normalizing each predictor's $\beta$ weight by the sum of the $\beta$ weights of the conditions involved in the comparison, resulting in values within a range of -1 and 1 . Thus, when two conditions, $a$ and $b$, were compared, the $\beta$ weight of $a$ was normalized by $a /(a$ $+b$ ); when three conditions were compared, the $\beta$ weight of $a$ was calculated as $a /(a+b+c)$. The normalized value of each predictor was then assigned to one of the three RGB color values of each map vertex. Thus, if a vertex was modulated mainly by one condition, this vertex would appear in the color assigned to this condition; if several conditions modulated the vertex, the color would appear as a mixture of the colors assigned to those conditions. Furthermore, all three RGB values were scaled according to the sum of the involved $\beta$ weights; thus, vertices with high $\beta$ weights (i.e., at which the experimental conditions modulated the BOLD signal comparatively much) were colored more intensely than vertices with low sums of the $\beta$ weights. Only vertices found active in the contrast of each respective condition against baseline were projected onto the cortical maps.

Regions of interest. To compare our results to previous findings, we complemented our gradient analysis with a region-of-interest (ROI) analysis. We created six ROIs around coordinates defined by previous studies (see below): visual area V7; two ROIs in the middle of the intraparietal sulcus (IPS1 and IPS2); two ROIs in anterior IPS (aIPS1 and aIPS2); and one ROI in the anterior precuneus $(\mathrm{aPCu})$. Each ROI comprised $\sim 300$ vertices centered around the Talairach coordinates of areas in left PPC. ROIs were analyzed only in the left hemisphere to account for the fact that participants used their right hand and foot (they obviously moved both eyes during saccades). In previous studies, ROIs V7, IPS1, and IPS2 were mapped using topographic mapping of saccades (i.e., their coordinates were functionally defined by eye movements) (Schluppeck et al., 2005; Silver et al., 2005; Hagler et al., 2007; Levy et al., 2007). V7 is located in Brodmann's area (BA) 19, whereas IPS1 and IPS2 lie anterior to $\mathrm{V} 7$ in BA 7. In contrast to these regions, ROI superior end of the parieto-occipital sulcus (sPOS) (Filimon et al., 2009) was defined by selecting voxels that were located between the sPOS and the subparietal sulcus, and were active when participants reached while their hand was visible; the coordinates of this sPOS area were very similar to those of IPS1, as defined by saccades, and were therefore collapsed with this area. ROI aPCu (Filimon et al., 2009) had the same functional criteria as sPOS, but was located between the POS and the cingulate sulcus in BA 7. ROI aIPS (Beurze et al., 2009) is located in BA 40 and was obtained by a conjunction of reach and saccade planning-related activation. Finally, ROI aIPS (Blangero et al., 2009) resulted as one of several reach-related areas from a meta-analysis of fMRI reaching studies and was chosen here for its anterior PPC location; it is located in BA 40, but very near to the hand area of primary somatosensory cortex (S1) and at the border toward BA 7.

When several studies reported the same area, we averaged the reported coordinates (for Talairach coordinates of all areas, see Table 1). When brain coordinates were given in Montreal Neurological Institute (MNI) space, Talairach coordinates were determined using the Nonlinear Yale MNI to Talairach Conversion Algorithm, as implemented online at http://www.bioimagesuite.org/Mni2Tal/index.html. Because our fMRI analysis was based on cortical reconstructions rather than volume space, the exact Talairach coordinates were not always part of our cortex model (values in Fig. 5, therefore, are not always the exact mean of the studies used to define the ROIs and coordinates reported). However, no center point was further than $5 \mathrm{~mm}$ in any direction from the (average) Talairach coordinates reported by previous studies. The mean $\beta$ weights were extracted for each condition and visualized (Fig. 5) by coloring the part of a ring corresponding to that condition's fraction of the total sum of the $\beta$ weights of hand, foot, and saccade planning in the cue 2 phase (corresponding to the values used for creating the gradient maps).

Psychophysiological interactions. Functional connectivity was assessed to complement to the contrast and gradient analyses using a psychophysiological interaction (PPI) approach (Friston et al., 1997). We deem these connectivity analyses exploratory as our experiment was not designed for this type of analysis, and statistical power is therefore expected to be low. Activations are thus displayed at the statistical cutoff value $p=0.05$ 


\begin{tabular}{|c|c|c|c|}
\hline References & $x$ & $y$ & $z$ \\
\hline \multicolumn{4}{|l|}{ V7 } \\
\hline Schluppeck et al., 2005 & -25 & -80 & 27 \\
\hline Beurze et al., 2007 & -21 & -79 & 19 \\
\hline Levy et al., 2007 & -26 & -81 & 22 \\
\hline \multicolumn{4}{|l|}{ IPS1/SPOS } \\
\hline Schluppeck et al., 2005 & -21 & -76 & 42 \\
\hline Silver et al., 2005 & -23 & -76 & 39 \\
\hline Hagler et al., 2007 & -20 & -69 & 43 \\
\hline Levy et al., 2007 & -20 & -79 & 34 \\
\hline \multicolumn{4}{|l|}{ IPS2 } \\
\hline Schluppeck et al., 2005 & -18 & -71 & 52 \\
\hline Silver et al., 2005 & -19 & -75 & 48 \\
\hline Hagler et al., 2007 & -19 & -64 & 51 \\
\hline Levy et al., 2007 & -20 & -74 & 44 \\
\hline \multicolumn{4}{|l|}{ alPS1 } \\
\hline Beurze et al., 2009 & -39 & -46 & 43 \\
\hline \multicolumn{4}{|l|}{ alPS2 } \\
\hline Blangero et al., 2009 & $-32^{a}$ & $-35^{a}$ & $57^{a}$ \\
\hline \multicolumn{4}{|l|}{$\mathrm{aPCu}$} \\
\hline Filimon et al., 2009 & $-9^{a}$ & $-50^{a}$ & $54^{a}$ \\
\hline
\end{tabular}

The term alPS has been used for different regions and is therefore used for two labels here. sPOS coordinates (Prado et al., 2005; Filimon et al., 2009) are very close to IPS1 and are therefore not indicated separately. ${ }^{a}$ Denotes that coordinates were reported in MNI space and were converted to Talairach coordinates here (see Materials and Methods for details).

uncorrected. The PPI was seeded in areas IPS1, IPS2, and V7 using the demeaned average BOLD time course from the ROIs defined above. The time course of the cue 2 phases (collapsing over left and right target trials, separately for hand- and foot-pointing trials) was convolved with the hemodynamic response function, demeaned, and multiplied with the seed time course of each ROI. The PPI was calculated in the context of the full GLM as specified above, effectively controlling for all main and nuisance effects of the experiment, supplemented with the seed time course as a further predictor.

\section{Results}

Twenty-three participants performed a goal-directed movement task with instructed delays. On each trial, the spatial goal of the movement and the effector used to respond were separately specified at two consecutive time points (Beurze et al., 2007, 2009) (for a detailed description of the paradigm, see Fig. 1). Targets were presented at one of four eccentricities in either the left or right visual field. In half of the trials, participants first received information about which effector to use (Fig. 1a, eye, right hand, or right foot- "effector cue"), and, after a random interval (2-5s, in ms steps, uniform distribution), the spatial target was presented ("target cue"). In the other half of trials, this presentation order was reversed (i.e., the target cue preceded the effector cue) (Fig. 1b). A third cue ("movement cue") was presented after another random interval (2-6s) to prompt participants to execute the prepared motor response.

\section{Behavioral measures}

Participants followed the instructions correctly in most trials (average error rate: $6.7 \%$ ). For the correct trials, mean RTs were 575 $\mathrm{ms}$ (SE $23 \mathrm{~ms}$ ) for saccades, $649 \mathrm{~ms}$ (SE $28 \mathrm{~ms}$ ) for hand pointing, and $634 \mathrm{~ms}$ (SE $26 \mathrm{~ms}$ ) for foot pointing and were comparable to previous studies (Beurze et al., 2007, 2009). RTs differed significantly between the three body parts (repeated-measures ANOVA, $\left.F_{(2,40)}=5.5, p=0.018\right)$. Two-tailed $t$ tests for repeated measurements between each pair of conditions revealed that saccade RTs were faster than both hand $\left(t_{(20)}=2.4, p=0.025\right)$ and foot $\left(t_{(20)}=2.6, p=0.016\right)$. In contrast, hand and foot RTs did not differ significantly $\left(t_{(22)}=1.3, p=0.21\right)$.

Correlations of target eccentricity with movement amplitude were calculated for the four targets of each target side (left and right) separately, as an analysis across target sides would lead to artificially high correlations. The correlation of target eccentricity with saccade amplitude was 0.70 (2 SE interval: 0.60-0.78; asymmetric intervals due to Fisher $Z$-transformation of correlation values) for leftward and 0.76 (2 SE interval: 0.69-0.81) for rightward targets; hand movement amplitude of 0.47 (2 SE interval: $0.35-0.57$ ) for leftward and 0.50 (2 SE interval: $0.42-0.58)$ for rightward targets; and foot movement amplitude 0.51 (2 SE interval: 0.43-0.59) for leftward and 0.50 (2 SE interval: 0.38-0.60) for rightward targets. An ANOVA with factors body part (eye, hand, foot) and target side (left, right) revealed only a main effect for body part $\left(F_{(2,40)}=19.8, p<0.001\right)$, but no main effect and interaction with target side (both $F<1, p>0.33$ ). Unsurprisingly, further ANOVAs, separately comparing each pair of body parts, showed that accuracy was higher for saccades than for hand and foot pointing (both main effects of body part: $F_{(1,20)}>21.9$, $p<0.001)$. However, performance did not differ between hand and foot pointing (main effect of body part: $F_{(1,22)}<1, p=0.64$ ).

\section{Functional MRI-hand versus eye movement planning}

We sought to validate our protocol against previous studies on movement planning that used similar fMRI paradigms and therefore first compared hand and eye movements. Our paradigm allowed distinguishing responses related to goal-directed movement plans from effector-specific activation in the absence of a known spatial goal (when the effector was cued first), as well as from later processing related to the actual movement of the effector and its subsequent sensory feedback (because movement execution was cued after a delay). Following the presentation of the second cue ("cue 2" from hereon), participants had received the information needed to fully specify the motor plan necessary to organize the upcoming pointing movement, because both the effector cue and the target cue had been presented. We contrasted cerebral activity evoked by eye and hand movement planning at this cue 2 stage against that evoked during a fixation baseline. Consistent with previous findings (Astafiev et al., 2003; Connolly et al., 2003; Medendorp et al., 2005; Beurze et al., 2007, 2009; Connolly et al., 2007; Hagler et al., 2007; Levy et al., 2007; Curtis and Connolly, 2008; Filimon et al., 2009), we found that planning of both eye and hand movements relied on a largely overlapping cerebral network, encompassing areas along the intraparietal sulcus and the superior parietal lobule (SPL; from the sPOS to the precuneus), as well as the supplementary motor area (SMA) and cingulate motor area (CMA), and dorsal and ventral premotor areas (Fig. 2a). Areas more active for hand movement planning, assessed with a contrast of hand versus eye movement planning (Fig. 2b), were centered around the hand area of the left primary motor cortex (M1), the CMA, and the very anterior part of the left SPL. Areas more active for saccade planning (assessed with a contrast of eye versus hand movement planning) were located mainly in occipital-parietal areas bilaterally, encompassing areas V7, IPS1, and IPS2, which have recently been shown to code target locations in topographic maps (Schluppeck et al., 2005; Silver et al., 2005; Hagler et al., 2007; Levy et al., 2007) (Table 1, Fig. $2 b$ ). However, both IPS1 and IPS2 were active also for hand movement planning, whereas V7 was activated exclusively for saccade planning (Fig. 2a). Some studies have reported handrelated activity in the sPOS (Prado et al., 2005; Filimon et al., 2009); the Talairach coordinates of this area are very near to those 
of what others have termed IPS1 (see Table 1), and, accordingly, there was both eye and hand activity in this area. To allow a more fine-grained comparison of hand and saccade planning, we created a gradient map (Levy et al., 2007; Stark and Zohary, 2008; Beurze et al., 2009), which directly compares the parameter estimates ( $\beta$ weights) of the GLM for hand and eye movement planning (Fig. 2c). Such maps give an unbiased view on the parameter estimates (i.e., here, the magnitudes of the eye and hand effects), rather than focusing on the rejection of null hypotheses (as done during statistical inference). The gradient map showed qualitatively similar results as the statistical contrasts, with a saccade bias in more posterior areas, a strong hand bias in anterior PPC and primary motor areas, and similar activity in intermediate PPC areas.

\section{Functional MRI- hand versus foot} movements: planning and execution To test whether the use of different limbs activated different brain areas in PPC, we compared the motor planning for hand and foot pointing. Figure $3 a$ shows the activation for each of the two movement planning conditions against the fixation baseline (cue 2 hand $>$ fixation baseline, cue 2 foot $>$ fixation baseline). This figure reveals a large overlap in all brain areas with the exception of the area around the central and postcentral sulci, mainly in the left hemisphere. Although Figure $3 a$ suggests some limb-specific activation to be present also in PPC, the contrast of hand and foot planning (Fig. $3 b$, cue 2 hand $>$ cue 2 foot) revealed differences almost exclusively in the primary and cingulate motor areas and in somatosensory cortex, as well as in the cortex adjacent to these areas. Hand and foot planning differed in the most anterior part of the SPL in BA 5, marginally extending into BA 7 (medially) and BA 40 (laterally). Here, hand activity was more lateral, and foot activity more medial, consistent with reports of differences between hand and foot motor imagery (Bakker et al., 2008). Importantly, no differences between hand and foot planning were found in more posterior PPC, including areas IPS1 and IPS2 and putative human PRR (Connolly et al., 2003). This indicates that large parts of PPC, and especially the regions located in and around the IPS, were similarly active for both limbs. We then compared activation differences for the two effectors during actual movement execution (hand movement $>$ foot movement). Figure $3 b$ illustrates that the result of this contrast was virtually identical to that of movement planning, suggesting that processing differences for hands and feet are restricted to those cortical areas that are classically labeled as motor and somatosensory areas. As a further test, we created a $\beta$ weight gradient map similar to the one used to compare hand and eye movement planning, but here comparing hand and foot movement planning (Fig. 3c). Confirming our statistical analyses, this map showed a strong hand bias around the M1/S1 hand region, and a strong foot bias around the M1/S1 foot region and in the postcentral SPL region in the left hemisphere, but no consistent biases for hand or foot planning were evident in PPC.

\section{Functional MRI-limb versus eye movement planning}

Next, we contrasted foot motor planning against saccade planning (in the cue 2 phase) with a contrast analogous to that used in the hand-eye comparison above (cue 2 eye $>$ cue 2 foot). The results of this contrast were surprisingly similar to the hand-eye comparison (Fig. 4a,b). To confirm this statistically, we calculated a conjunction between the two contrasts (Fig. $4 c$, conjunction of cue 2 hand $>$ cue 2 eye and cue 2 foot $>$ cue 2 eye). The results were very similar to the individual contrasts (Fig. $4 a, b$ ) and revealed that the more posterior areas of PPC were more active for eye movement than for hand and foot motor planning; this included areas IPS1 and IPS2. In fact, IPS2 was the most anterior region active in this contrast. The overlap between planning-related activity of hand and foot movements cannot reflect floor effects, as participants executed spatially accurate goal-directed actions rather than stereotyped movements throughout the experiment. Next, we tested whether the saccaderelated activation in these contrasts indicated merely a bias (i.e., that these areas were more, but not exclusively, active during saccade planning) or specificity (i.e., that these areas were active only during saccade, but not during hand and foot planning). To do so, we calculated a conjunction between hand and foot-related activation, each against the fixation baseline (cue 2 hand $>$ fixation and cue 2 foot $>$ fixation), and overlaid the resulting map with the saccade-related activation map (Fig. $4 d$ ). This comparison revealed that the more posterior regions (including V7) were indeed specific for saccade planning. In contrast, both IPS1 and IPS2 were biased for saccade planning, but also showed activation during both hand and foot planning. In a further examination, we created a $\beta$ weight gradient map for eye versus foot movement planning (Fig. 4e) like the gradient map for eye versus hand movement planning (Fig. 2c). Both maps showed a strong bias for hand and foot, respectively, around their M1/S1 and anterior SPL regions. In contrast, the gradient maps showed a bias for saccade planning along the IPS, both compared with hand and foot planning. To summarize the results from the different statistical anal- 

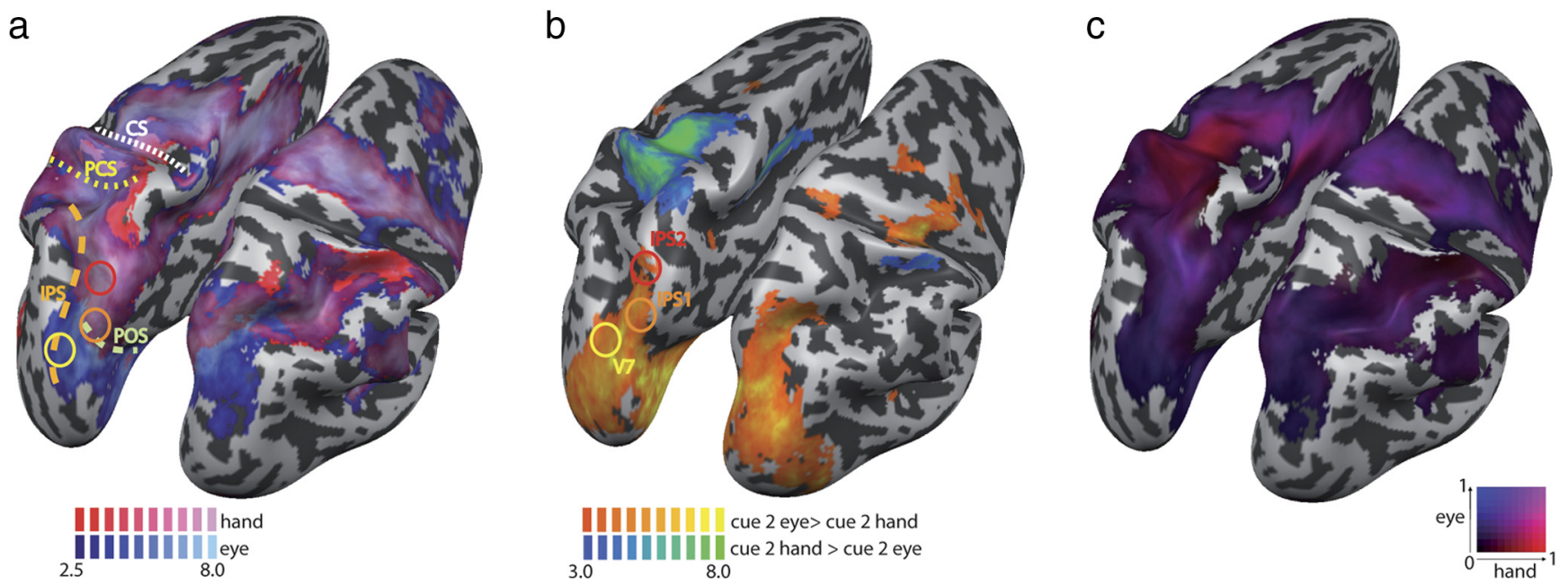

Figure 2. Eye and hand planning in the cue 2 phase (both effector and visual target known to participant). $\boldsymbol{a}$, Eye movement planning (blue) and hand planning (red), each against fixation baseline. The yellow, orange, and red circles indicate the approximate locations of V7, IPS1, and IPS2, respectively. Hand movement planning exclusively activates the hand region of M1/S1 and some anterior SPL regions; eye movement planning exclusively activates occipitoparietal regions, for example, area V7. Dashed lines denote major sulci. CS, Central sulcus; PCS, postcentral sulcus. $\boldsymbol{b}$, Contrast of hand $>$ eye movement planning (blue) and eye $>$ hand movement planning (yellow). Hand-biased regions are located in anterior SPL; saccade-biased regions are located in the posterior end of PPC. Circles as in $\boldsymbol{a}$. All activations are FDR $(p<0.05)$ corrected; $t$ values are indicated in the color legends. $\boldsymbol{c}$, Gradient map of saccade versus hand movement planning (cue 2). At each vertex (equivalent to a voxel, but represents a location only on the reconstructed cortical surface), the $\beta$ weight from the GLM is compared for the two involved movement planning conditions. The lighter the color, the larger is the sum of the involved $\beta$ weights at this vertex (indicating a strong modulation by the experimental conditions). The map is restricted to those vertices that were active in either of the involved conditions (each contrasted against fixation baseline). There is a strong bias for hand planning around the M1/S1 area, extending into the anterior SPL. Along the IPS, both eye and hand movement planning evoke activation.

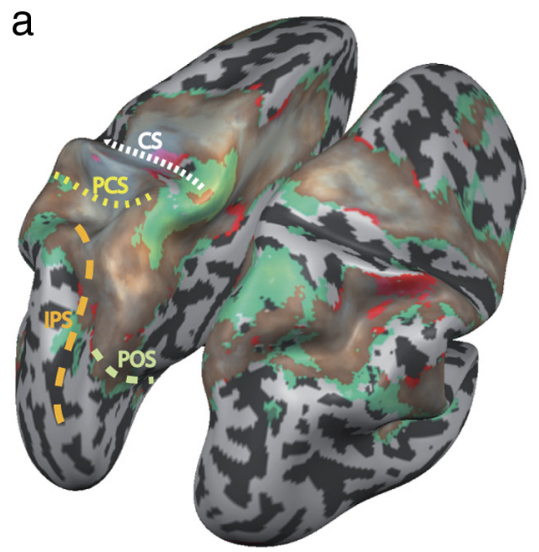

IIIIIIIII cue 2 hand $>$ baseline |||||||||| cue 2 foot $>$ baseline b

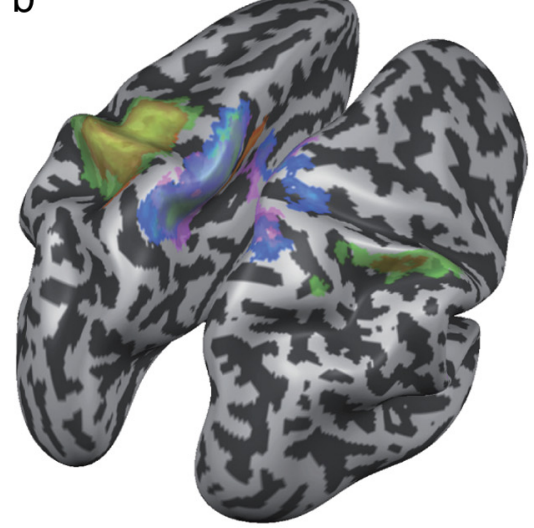

|| || || || cue 2 foot $>$ cue 2 hand IIIIII cue 2 hand $>$ cue 2 foot

$$
\begin{aligned}
& 3.2 \\
& \text { move foot }>\text { move hand } \\
& \text { move hand }>\text { move foot }
\end{aligned}
$$

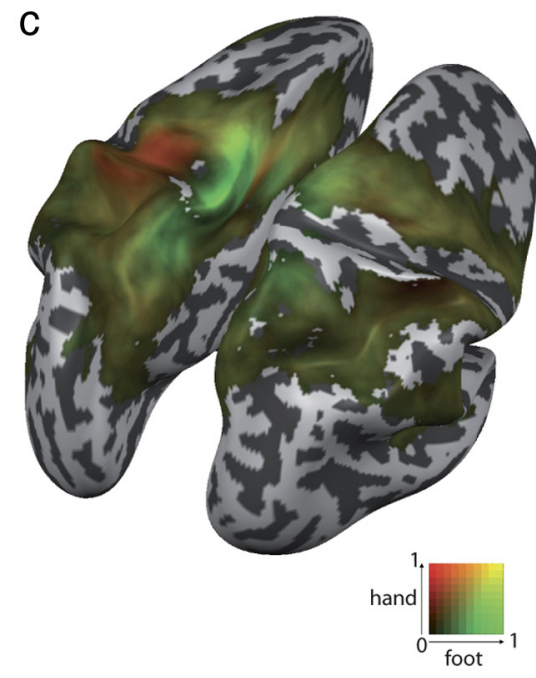

C

Figure 3. Hand and foot movement planning. $\boldsymbol{a}$, Comparison of hand (red) and foot (green) movement planning, each against fixation baseline. The two activations largely overlap, with the exception of the M1/S1 areas. Dashed lines denote major sulci. CS, Central sulcus; PCS, postcentral sulcus. $\boldsymbol{b}$, Comparison of movement planning (cue 2 phase) and movement execution. Contrast cue 2 hand (yellow) $>$ cue 2 foot (blue) is overlaid with contrast move hand (green) $>$ move foot (pink). The two contrasts largely overlap, indicating that differential processing for movement planning is mediated by those areas that also mediate movement execution. $c$, Gradient map of hand versus foot movement planning. The only areas showing a bias are the M1/S1 areas. In PPC, no bias toward either effector is evident.

yses and gradient maps, we created a map in which the $\beta$ weights during the cue 2 phase of all three effectors, eye, hand, and foot, were compared with each other (Fig. 5). This figure also shows the relative contribution of each effector to the different areas that have been previously identified.

\section{Functional fMRI-psychophysiological interactions}

We complemented the contrast analyses by functional connectivity analysis. This approach statistically tests the interaction between a psychological variable (here, the cue 2 phases of hand and foot pointing, respectively) and the BOLD signal of a seed region (here, IPS1, IPS2, and V7, respectively) at each vertex of the reconstructed cortical surface. Because contrasts and gradient analysis showed similar activation in areas IPS1 and IPS2 for both hand and foot pointing, we hypothesized that these ROIs should exhibit functional connectivity with more lateral regions of motor cortex and anterior PPC during hand-pointing trials, and with more medial regions during foot-pointing trials. V7, in contrast, should show little or no connectivity with hand and foot motor regions or anterior PPC during pointing trials, as V7 


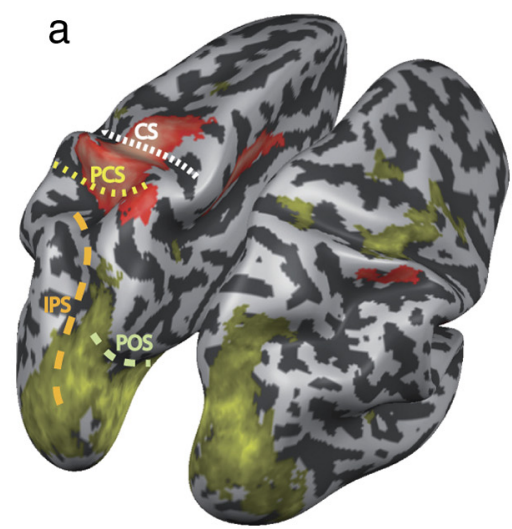

cue 2 hand $>$ cue 2 eye
cue 2 eye $>$ cue 2 hand b

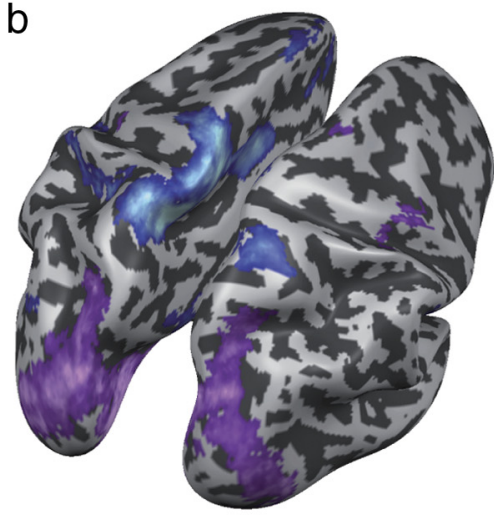

cue 2 foot $>$ cue 2 eye
cue 2 eye $>$ cue 2 foot
C

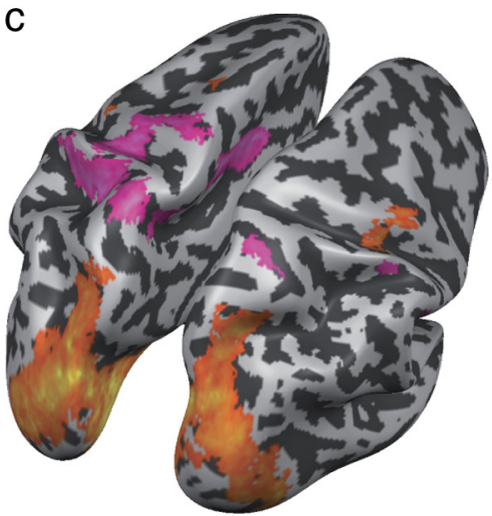

cue 2 hand $>$ cue 2 eye AND 1 11 1 cue 2 foot $>$ cue 2 eye cue 2 eye $>$ cue 2 hand AND 3.0 8.0

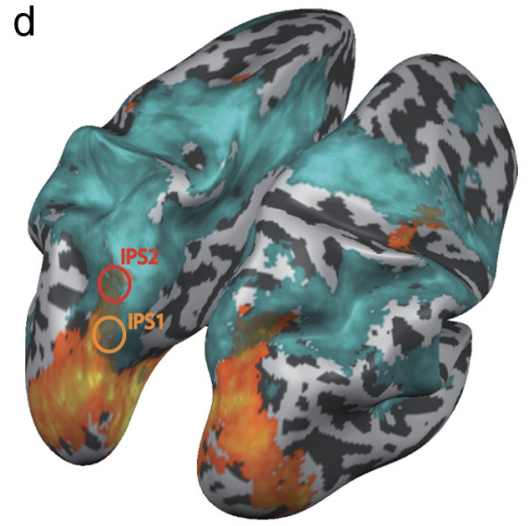

3.0 (saccade > hand) AND (saccade > foot)
(hand $>$ baseline) AND (foot $>$ baseline) e

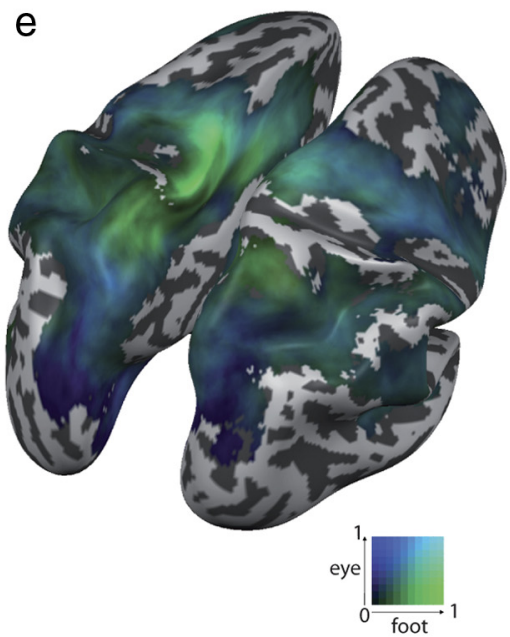

Figure 4. Comparison of eye with hand/foot activity. $\boldsymbol{a}$, Contrast cue 2 eye (yellow) $>$ cue 2 hand (red). $\boldsymbol{b}$, Contrast cue 2 eye (purple) $>$ cue 2 foot (blue). $\boldsymbol{c}$, Conjunction of the two contrasts (eye: orange; hand/foot: pink). Eye-biased activation is virtually identical in all comparisons. $d$, Comparison of hand and foot movement planning with eye movement planning. The common activation of hand and foot movement (conjunction of cue 2 hand and cue 2 foot, both against baseline, cyan) is overlaid with eye-related activation (conjunction of cue 2 eye $>$ cue 2 hand and cue 2 eye $>$ cue 2 foot, yellow). The eye-related activation overlaps with hand and foot activation in region IPS1 (yellow circle) and IPS2 (red circle), indicating biased, but not exclusive, processing for eye movements in these areas. The $t$ values are indicated in the color legends of each subfigure. $\boldsymbol{e}$, Gradient map of saccade versus foot movement planning. The biases are very similar to those of the eye versus hand comparison.

showed high activity during saccade trials only. We consider the connectivity analyses exploratory (see Materials and Methods), and results are presented in Figure 6, $a$ (hand pointing) and $b$ (foot pointing), with uncorrected $p$ values. During hand pointing, connectivity of IPS1, IPS2, and V7 overlap, spreading over lateral regions of postcentral sulcus, central sulcus, premotor areas, and SMA. IPS1 and IPS2, however, show more widespread connectivity, especially in all motor regions. More importantly, the most significant part of the M1 hand activation in the contrast analyses (Fig. 6a, circled yellow) showed connectivity to IPS1 and IPS2, but not to V7. During foot pointing, IPS1 and IPS2 showed connectivity with medial motor, premotor, and supplementary motor regions, including the most significant $\mathrm{M} 1$ region of the contrast analyses (Fig. 6b, circled green), whereas no connectivity was observed for V7. Thus, the IPS1 and IPS2, which code a common representation of hand and foot spatial actions, connect to the effector-specific regions over the motor strip in a functional manner, with the strength of the connection depending on the type of trial (foot or hand) that is to be executed. The lack of postcentral connectivity may be due to the relatively high ana- tomical variance in SPL regions (Scheperjans et al., 2008), which might lead to sensitivity loss of the already weakly powered PPI analysis in this region despite the cortical alignment methods used for statistical testing.

\section{Functional MRI-effector versus spatial responses}

The analyses so far have looked at those stages of the trial during which both effector (eye, hand, foot) and the visual target location were known to the participants (i.e., at the cue 2 phase of our trials), as well as during motor execution stage. In the first cueing phase of each trial (the cue 1 phase), participants received only partial information, either the effector to be used for the upcoming movement or the target location of the movement. We compared activity in the cue 1 phase for these two types of trials to investigate whether there were areas that prepare movements for a specific effector in the absence of a target. To this end, we first contrasted each pair of effectors for trials in which the effector was revealed in the cue 1 phase (cue 1 eye $>$ cue 1 hand; cue 1 eye $>$ cue 1 foot; cue 1 hand $>$ cue 1 foot). None of these contrasts revealed any activation in PPC. We then calculated a con- 


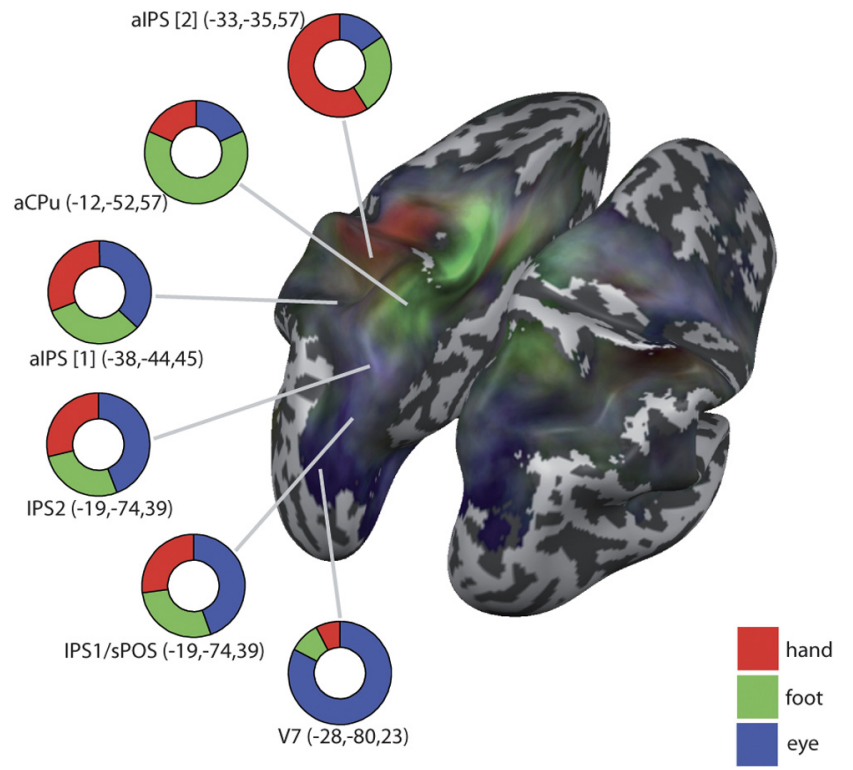

Figure 5. Comparison of all three effectors (eye, hand, foot). The gradient map shows the relation of the $\beta$ weights of eye (blue), hand (red), and foot (green) planning during the cue 2 phase. Lightness of the colors indicates the overall sum of the $\beta$ weights, with lighter colors indicating higher $\beta$ values (i.e., "stronger" activation). Each ring visualizes the distribution of $\beta$ weights for the eye, hand, and foot conditions. Center coordinates (given in Talairach coordinates) for the indicated areas were chosen according to previous reports (see Materials and Methods; Table 1).
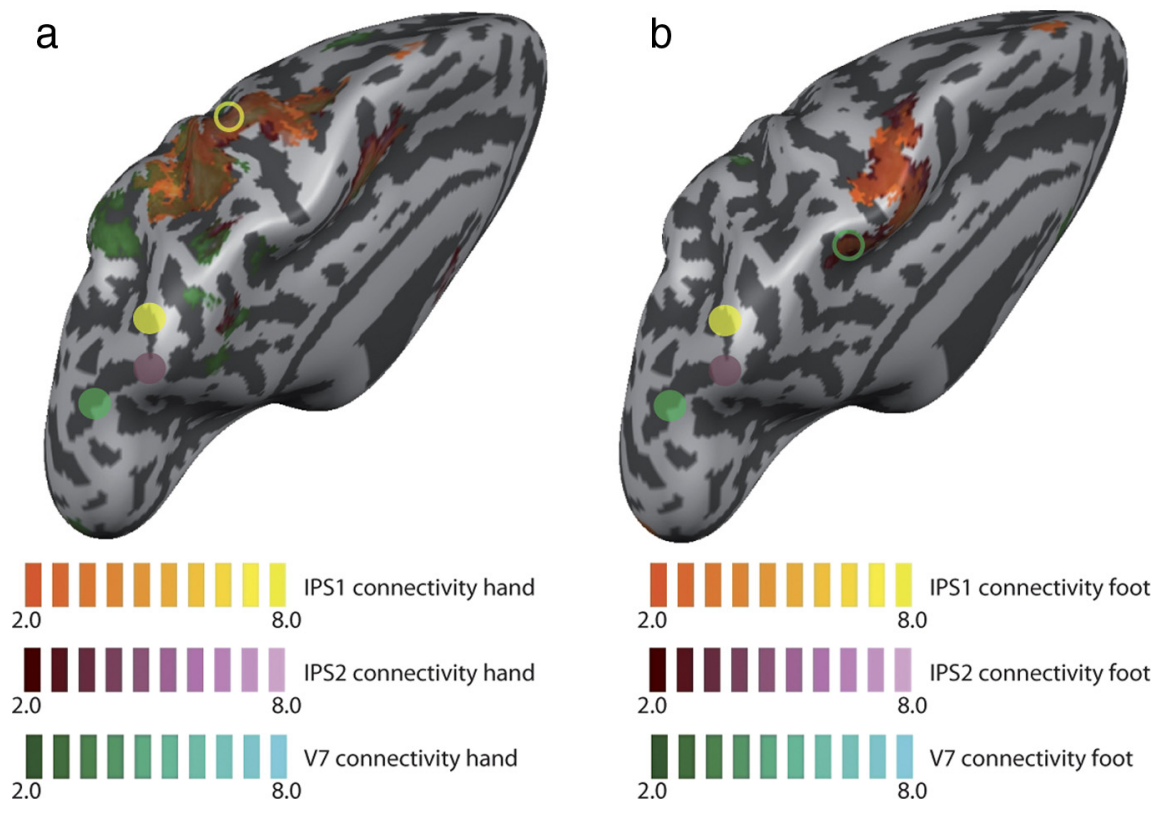

Figure 6. Functional connectivity of PPC regions. $\boldsymbol{a}, \boldsymbol{b}$, Results for connectivity during hand trials are shown in $\boldsymbol{a}$, and during foot trials in $\boldsymbol{b}$. Activity for seed regions IPS1, IPS2, and V7 are overlaid to allow comparison. Seed regions are indicated by filled colored circles (yellow, IPS1; red, IPS2; green, V7) and are identical for hand and foot analyses. The open circles show the most active region during hand ( $\boldsymbol{a}$, yellow) and foot ( $\boldsymbol{b}$, green) movement planning in the GLM contrasts shown in Figure $4 a, b$. No connectivity is observed for V7 (green shading in $\boldsymbol{b}$ ) during foot planning.

trast in which all three types of effector cues were contrasted with target presentation $[$ (cue 1 eye + cue 1 hand + cue 1 foot $) / 3>$ cue 1 target]. No activation was seen in PPC, which was higher for effector than for target processing. Rather, there was widespread activation in PPC for target processing over effector processing, predominantly in the left hemisphere when thresholding with the false discovery rate (Fig. 7). When using an exploratory threshold of $p=0.05$ (uncorrected), widespread activation was seen also in the right PPC (Fig. 7).

\section{Discussion}

The current study tested whether motor planning in PPC is divided into different regions according to a somatotopic, effectorspecific arrangement beyond the well known biases for eye versus hand movement planning. Our main result is that PPC responses during the planning of hand and foot movements were statistically indistinguishable, whereas hand- and foot-related responses were spatially segregated from those evoked during the planning of eye movements to the same visual targets. Importantly, whereas the lack of activation in the direct contrast between hand and foot movement planning may be interpreted as a null result, this is not the case for the contrasts of hand versus eye and foot versus eye movement planning, respectively. Furthermore, the fact that the conjunction of these two contrasts revealed virtually identical results as each of the single contrasts strengthens this result. Connectivity analyses further confirm overlapping processing in PPC for hand and foot pointing, as connectivity from IPS1 and IPS2 regions was with lateral, hand-related motor regions during hand trials, and with medial, foot-related regions during foot trials. Thus, our results suggest a functional rather than effector-specific organization of human posterior parietal cortex.

Large-scale organization of PPC encompassing different types of limbs, as shown here, is compatible with a number of other recent findings. First, neurons in area PEc (the caudal part of area 5, located on the SPL and neighboring the IPS) were reported to be responsive to tactile stimulation and joint rotation of both upper and lower limbs (Breveglieri et al., 2006), with anatomical connections to foot and hand motor cortex and premotor cortex (Bakola et al., 2010). Second, a recent study on motor observation implies that, rather than type of limb, it is the type of action that determines regional organization in PPC (Jastorff et al., 2010). Third, such organization would be consistent with the affordance competition hypothesis, which states that different possible actions are active in parallel, competing against each other until one is selected and executed (Cisek, 2007).

The eyes play an entirely different role than the limbs in that they are used to explore the environment by aligning the fovea with objects of interest in the environment. It is hardly surprising that planning saccadic movements recruits brain regions located relatively early in the visual cortical pathway. In contrast, other body parts are used to act upon objects in the environment. Here, we show that the most anterior portion of the SPL, namely BA 5, has a lateral-tomedial gradient for hand versus foot movements, although there is considerable overlap for the two limbs even in this area (Fig. $3 c$ ). Area 5 encroaches on primary somatosensory areas (Penfield and Boldrey, 1937), which display fine-grained somatotopy. This 

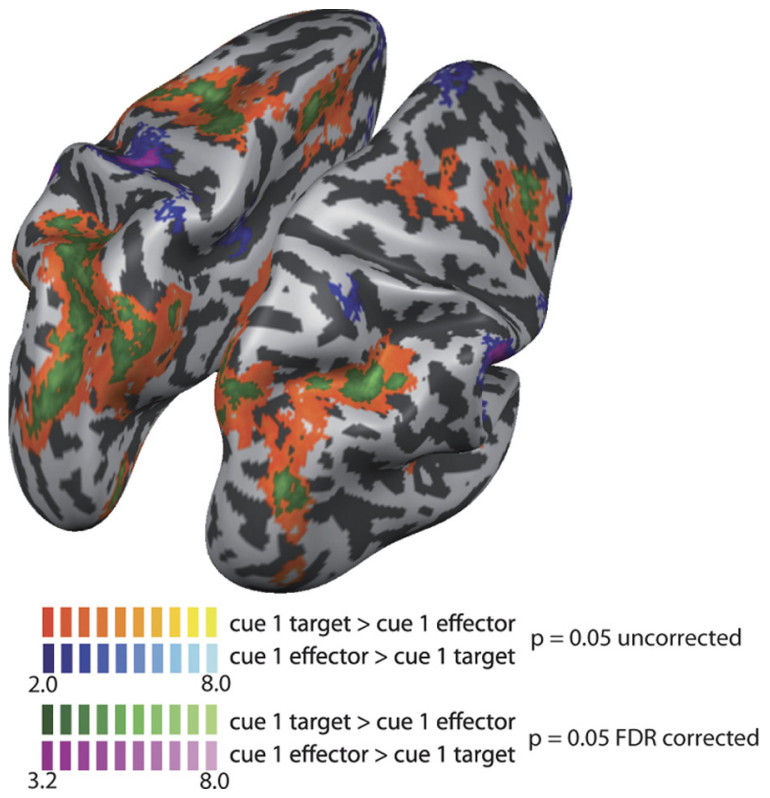

Figure 7. Comparison of target versus effector activity. PPC activation during the cue 1 phase, in which only either effector or spatial target information was known. Shown is the contrast of cue 1 target $>$ (cue 1 eye + cue 1 hand + cue 1 foot)/3. Green colors represent FDR corrected activation, and orange colors uncorrected $(p=0.05)$ activation. The $t$ values are indicated in the color legend.

portion of the SPL could therefore specify the effector-specific motor plan together with frontal motor areas, including M1 and the premotor cortex (Caminiti et al., 2010).

Computationally, one of the most important requirements that hand and foot pointing add to the requirements of making a saccade is the calculation of the spatial relationship between limb and target location. This calculation must be based on an estimate of the body's current posture and requires an alignment of the reference frames involved in visual processing and limb movement planning. Whereas saccade planning is thought to occur in eye-centered coordinates, both eye- and body-related reference frames are probably used for limb movement planning (Pesaran et al., 2006; Chang and Snyder, 2007; Marzocchi et al., 2008; Chang et al., 2009; Ferraina et al., 2009; McGuire and Sabes, 2009; Bernier and Grafton, 2010; Chang and Snyder, 2010; Pesaran et al., 2010). The PPC, and especially the SPL, has been suggested to play a major role in the estimation of the current limb positions using proprioceptive and visual information (Graziano et al., 2000; Shadmehr and Krakauer, 2008; Jackson et al., 2009), implicated, for example, by patients suffering from optic ataxia (Karnath and Perenin, 2005). In monkeys, area 5 neurons integrate vision and proprioception to represent limb posture (Graziano et al., 2000), and neurons in area PEc code for complex body postures involving not only hands and arms, but also legs and feet (Breveglieri et al., 2006, 2008).

Reaching to visual targets without vision of the hand activates the anterior reaching-related PPC areas more than posterior ones (Filimon et al., 2009). In fact, SPL is active also during nonvisual tasks, as when reaching toward one's own body parts in the dark (Pellijeff et al., 2006). Furthermore, congenitally blind and sighted people show similar SPL activation during tasks that require the exploration of an object with the hand (Fiehler et al., 2009). Body coding in SPL can thus be independent of vision, corroborating the present results of only little SPL activity during saccade planning. In this framework, our results suggest that PPC transforms different coordinate systems to relate different body parts to the (visual) target without any obvious spatial segregation in the neuronal populations supporting each effector. Our connectivity results suggest that the specific planning for the chosen body part is then mediated by increased connectivity with frontal motor areas like M1 and the premotor cortex. The specific form of clustering body versus eye movement planning may be due to the need to minimize axonal wiring across neuronal populations involved in movements of the body, as has recently been suggested analogously for frontal motor regions (Graziano and Aflalo, 2007; Meier et al., 2008).

Previous studies have demonstrated a caudo-rostral gradient in PPC responses when comparing movement planning for saccades and hand movements in monkeys (Burnod et al., 1999; Caminiti et al., 2010) and humans (Astafiev et al., 2003; Connolly et al., 2003; Medendorp et al., 2005; Beurze et al., 2007, 2009; Connolly et al., 2007; Filimon et al., 2009). The current study confirms those findings, but also indicates that they cannot be interpreted as evidence for effector-specific, somatotopic organization of PPC (Chang et al., 2008; Andersen and Cui, 2009). Other studies in monkeys (Breveglieri et al., 2008; Chang et al., 2008) and humans (Medendorp et al., 2005; Beurze et al., 2009; Blangero et al., 2009) have investigated effector specificity by comparing movement planning for the left and right hands, rather than by comparing eyes and hands. Differential PPC activity for movements involving these limbs was interpreted as indicating effector specificity in PPC. However, this comparison confounds possible effector specificity with hemispheric processing. Contralateral processing is one of the most prominent features of brain organization in both sensory and motor domains. Processing differences for the two hands may therefore not imply effector specificity, but rather a bias of each hemisphere in mediating the planning for the contralateral body. This account predicts that activity in PPC should be sensitive to the use of left and right limbs, but - as reported here-not to the type of limb of the same body side. In line with these considerations, ataxia can affect both limbs of the same body side while sparing those of the other body side (Rondot et al., 1977).

\section{Limitations of the present study}

Our results are constrained by the spatial resolution of our functional measurements and the averaging over participants typical for fMRI analysis. However, putative human homologs of monkey areas like LIP and PRR of considerably larger extent than one fMRI voxel have been reported (Grefkes and Fink, 2005; Orban et al., 2006). Furthermore, we minimized effects of anatomical variability by using landmark-oriented alignment methods (Fischl et al., 1999; van Atteveldt et al., 2004) (see Materials and Methods), maximizing the power to find limb-specific areas. Examination of single participants did not reveal any consistent pattern of hand- and foot-biased regions in our data.

Our functional considerations remain speculative, as the aim of the current experiment was to test for effector specificity in PPC, but did not address all possible differences between eye and limb movement planning. Yet, important alternative explanations of our data pattern were controlled for. Differences between eyes and limbs cannot reflect differences in difficulty of task or muscular control between eyes and limbs as eye-related activity was higher than limb activity in the more posterior PPC areas. Furthermore, differences between eye and limb planning cannot reflect attentional differences, as task presentation and design were identical throughout the experiment. Finally, it has been suggested that pointing tasks differ from true reaching tasks (Cul- 
ham et al., 2006; Cavina-Pratesi et al., 2010); therefore, reaching or grasping tasks may reveal further differences between hand and foot planning than those observed in the current study. However, because large limb movements interfere with MR acquisition (Culham et al., 2006), this possibility might require the use of other neuroimaging techniques.

\section{Conclusion}

To conclude, we report that PPC is organized according to a caudo-rostral visual-to-somatic gradient in PPC (Burnod et al., 1999; Beurze et al., 2007, 2009; Levy et al., 2007; Caminiti et al., 2010). Contrary to common view, this gradient does not seem to reflect effector specificity beyond a crude distinction between eye movements and movements of other body parts. Rather, activity in PPC related to different limbs is surprisingly uniform.

\section{References}

Andersen RA, Cui H (2009) Intention, action planning, and decision making in parietal-frontal circuits. Neuron 63:568-583.

Astafiev SV, Shulman GL, Stanley CM, Snyder AZ, Van Essen DC, Corbetta M (2003) Functional organization of human intraparietal and frontal cortex for attending, looking, and pointing. J Neurosci 23:4689-4699.

Bakker M, De Lange FP, Helmich RC, Scheeringa R, Bloem BR, Toni I (2008) Cerebral correlates of motor imagery of normal and precision gait. Neuroimage 41:998-1010.

Bakola S, Gamberini M, Passarelli L, Fattori P, Galletti C (2010) Cortical connections of parietal field PEc in the macaque: linking vision and somatic sensation for the control of limb action. Cereb Cortex 20:2592-2604.

Bernier PM, Grafton ST (2010) Human posterior parietal cortex flexibly determines reference frames for reaching based on sensory context. Neuron 68:776-788.

Beurze SM, Van Pelt S, Medendorp WP (2006) Behavioral reference frames for planning human reaching movements. J Neurophysiol 96:352-362.

Beurze SM, de Lange FP, Toni I, Medendorp WP (2007) Integration of target and effector information in the human brain during reach planning. J Neurophysiol 97:188-199.

Beurze SM, de Lange FP, Toni I, Medendorp WP (2009) Spatial and effector processing in the human parietofrontal network for reaches and saccades. J Neurophysiol 101:3053-3062.

Blangero A, Menz MM, McNamara A, Binkofski F (2009) Parietal modules for reaching. Neuropsychologia 47:1500-1507.

Breveglieri R, Galletti C, Gamberini M, Passarelli L, Fattori P (2006) Somatosensory cells in area PEc of macaque posterior parietal cortex. J Neurosci 26:3679-3684.

Breveglieri R, Galletti C, Monaco S, Fattori P (2008) Visual, somatosensory, and bimodal activities in the macaque parietal area PEc. Cereb Cortex 18:806-816.

Brown MR, Vilis T, Everling S (2008) Isolation of saccade inhibition processes: rapid event-related fMRI of saccades and nogo trials. Neuroimage 39:793-804.

Buneo CA, Jarvis MR, Batista AP, Andersen RA (2002) Direct visuomotor transformations for reaching. Nature 416:632-636.

Burnod Y, Baraduc P, Battaglia-Mayer A, Guigon E, Koechlin E, Ferraina S, Lacquaniti F, Caminiti R (1999) Parieto-frontal coding of reaching: an integrated framework. Exp Brain Res 129:325-346.

Calton JL, Dickinson AR, Snyder LH (2002) Non-spatial, motor-specific activation in posterior parietal cortex. Nat Neurosci 5:580-588.

Caminiti R, Chafee MV, Battaglia-Mayer A, Averbeck BB, Crowe DA, Georgopoulos AP (2010) Understanding the parietal lobe syndrome from a neurophysiological and evolutionary perspective. Eur J Neurosci 31:2320-2340.

Cavina-Pratesi C, Monaco S, Fattori P, Galletti C, McAdam TD, Quinlan DJ, Goodale MA, Culham JC (2010) Functional magnetic resonance imaging reveals the neural substrates of arm transport and grip formation in reach-to-grasp actions in humans. J Neurosci 30:10306-10323.

Chang SW, Snyder LH (2007) Monkey PRR uses an arm-centered frame of reference while executing contralateral but not ipsilateral arm movements. Program No 281172007 Neuroscience Meeting Planner.

Chang SW, Snyder LH (2010) Idiosyncratic and systematic aspects of spatial representations in the macaque parietal cortex. Proc Natl Acad Sci U S A 107:7951-7956

Chang SW, Dickinson AR, Snyder LH (2008) Limb-specific representation for reaching in the posterior parietal cortex. J Neurosci 28:6128-6140.

Chang SW, Papadimitriou C, Snyder LH (2009) Using a compound gain field to compute a reach plan. Neuron 64:744-755.

Cisek P (2007) Cortical mechanisms of action selection: the affordance competition hypothesis. Philos Trans R Soc Lond B Biol Sci 362:1585-1599.

Connolly JD, Andersen RA, Goodale MA (2003) FMRI evidence for a "parietal reach region" in the human brain. Exp Brain Res 153:140-145.

Connolly JD, Goodale MA, Cant JS, Munoz DP (2007) Effector-specific fields for motor preparation in the human frontal cortex. Neuroimage 34:1209-1219.

Cui H, Andersen RA (2007) Posterior parietal cortex encodes autonomously selected motor plans. Neuron 56:552-559.

Culham JC, Cavina-Pratesi C, Singhal A (2006) The role of parietal cortex in visuomotor control: what have we learned from neuroimaging? Neuropsychologia 44:2668-2684.

Curtis CE, Connolly JD (2008) Saccade preparation signals in the human frontal and parietal cortices. J Neurophysiol 99:133-145.

Diedrichsen J, Hashambhoy Y, Rane T, Shadmehr R (2005) Neural correlates of reach errors. J Neurosci 25:9919-9931.

Ferraina S, Brunamonti E, Giusti MA, Costa S, Genovesio A, Caminiti R (2009) Reaching in depth: hand position dominates over binocular eye position in the rostral superior parietal lobule. J Neurosci 29:11461-11470.

Fiehler K, Burke M, Bien S, Röder B, Rösler F (2009) The human dorsal action control system develops in the absence of vision. Cereb Cortex 19:1-12.

Filimon F, Nelson JD, Huang RS, Sereno MI (2009) Multiple parietal reach regions in humans: cortical representations for visual and proprioceptive feedback during on-line reaching. J Neurosci 29:2961-2971.

Fischl B, Sereno MI, Tootell RB, Dale AM (1999) High-resolution intersubject averaging and a coordinate system for the cortical surface. Hum Brain Mapp 8:272-284.

Friston KJ, Buechel C, Fink GR, Morris J, Rolls E, Dolan RJ (1997) Psychophysiological and modulatory interactions in neuroimaging. Neuroimage 6:218-229.

Goebel R, Esposito F, Formisano E (2006) Analysis of functional image analysis contest (FIAC) data with brainvoyager QX: from single-subject to cortically aligned group general linear model analysis and self-organizing group independent component analysis. Hum Brain Mapp 27:392-401.

Graziano MS, Aflalo TN (2007) Mapping behavioral repertoire onto the cortex. Neuron 56:239-251.

Graziano MS, Gross CG (1998) Spatial maps for the control of movement. Curr Opin Neurobiol 8:195-201.

Graziano MS, Hu XT, Gross CG (1997) Visuospatial properties of ventral premotor cortex. J Neurophysiol 77:2268-2292.

Graziano MS, Cooke DF, Taylor CS (2000) Coding the location of the arm by sight. Science 290:1782-1786.

Grefkes C, Fink GR (2005) The functional organization of the intraparietal sulcus in humans and monkeys. J Anat 207:3-17.

Hagler DJ Jr, Riecke L, Sereno MI (2007) Parietal and superior frontal visuospatial maps activated by pointing and saccades. Neuroimage 35:1562-1577.

Hinkley LB, Krubitzer LA, Padberg J, Disbrow EA (2009) Visual-manual exploration and posterior parietal cortex in humans. J Neurophysiol 102:3433-3446.

Hoshi E, Tanji J (2000) Integration of target and body-part information in the premotor cortex when planning action. Nature 408:466-470.

Hyvärinen J (1981) Regional distribution of functions in parietal association area 7 of the monkey. Brain Res 206:287-303.

Jackson SR, Newport R, Husain M, Fowlie JE, O'Donoghue M, Bajaj N (2009) There may be more to reaching than meets the eye: re-thinking optic ataxia. Neuropsychologia 47:1397-1408.

Jastorff J, Begliomini C, Fabbri-Destro M, Rizzolatti G, Orban GA (2010) Coding observed motor acts: different organizational principles in the parietal and premotor cortex of humans. J Neurophysiol 104:128-140.

Karnath HO, Perenin MT (2005) Cortical control of visually guided reaching: evidence from patients with optic ataxia. Cereb Cortex 15:1561-1569. 
Levy I, Schluppeck D, Heeger DJ, Glimcher PW (2007) Specificity of human cortical areas for reaches and saccades. J Neurosci 27:4687-4696.

Marzocchi N, Breveglieri R, Galletti C, Fattori P (2008) Reaching activity in parietal area V6A of macaque: eye influence on arm activity or retinocentric coding of reaching movements? Eur J Neurosci 27:775-789.

McGuire LM, Sabes PN (2009) Sensory transformations and the use of multiple reference frames for reach planning. Nat Neurosci 12:1056-1061.

Medendorp WP, Goltz HC, Crawford JD, Vilis T (2005) Integration of target and effector information in human posterior parietal cortex for the planning of action. J Neurophysiol 93:954-962.

Meier JD, Aflalo TN, Kastner S, Graziano MS (2008) Complex organization of human primary motor cortex: a high-resolution fMRI study. J Neurophysiol 100:1800-1812.

Murray EA, Coulter JD (1981) Organization of corticospinal neurons in the monkey. J Comp Neurol 195:339-365.

Orban GA, Claeys K, Nelissen K, Smans R, Sunaert S, Todd JT, Wardak C, Durand JB, Vanduffel W (2006) Mapping the parietal cortex of human and non-human primates. Neuropsychologia 44:2647-2667.

Pellijeff A, Bonilha L, Morgan PS, McKenzie K, Jackson SR (2006) Parietal updating of limb posture: an event-related fMRI study. Neuropsychologia 44:2685-2690.

Penfield W, Boldrey E (1937) Somatic motor and sensory representation in the cerebral cortex of man as studied by electrical stimulation. Brain 60:389-443.

Pesaran B, Nelson MJ, Andersen RA (2006) Dorsal premotor neurons encode the relative position of the hand, eye, and goal during reach planning. Neuron 51:125-134.
Pesaran B, Nelson MJ, Andersen RA (2010) A relative position code for saccades in dorsal premotor cortex. J Neurosci 30:6527-6537.

Prado J, Clavagnier S, Otzenberger H, Scheiber C, Kennedy H, Perenin MT (2005) Two cortical systems for reaching in central and peripheral vision. Neuron 48:849-858.

Rondot P, de Recondo J, Dumas JL (1977) Visuomotor ataxia. Brain 100:355-376.

Scheperjans F, Eickhoff SB, Hömke L, Mohlberg H, Hermann K, Amunts K, Zilles K (2008) Probabilistic maps, morphometry, and variability of cytoarchitectonic areas in the human superior parietal cortex. Cereb Cortex 18:2141-2157.

Schluppeck D, Glimcher P, Heeger DJ (2005) Topographic organization for delayed saccades in human posterior parietal cortex. J Neurophysiol 94:1372-1384.

Shadmehr R, Krakauer JW (2008) A computational neuroanatomy for motor control. Exp Brain Res 185:359-381.

Silver MA, Ress D, Heeger DJ (2005) Topographic maps of visual spatial attention in human parietal cortex. J Neurophysiol 94:1358-1371.

Snyder LH, Batista AP, Andersen RA (1997) Coding of intention in the posterior parietal cortex. Nature 386:167-170.

Stark A, Zohary E (2008) Parietal mapping of visuomotor transformations during human tool grasping. Cereb Cortex 18:2358-2368.

van Atteveldt N, Formisano E, Goebel R, Blomert L (2004) Integration of letters and speech sounds in the human brain. Neuron 43:271-282.

Van Der Werf J, Jensen O, Fries P, Medendorp WP (2010) Neuronal synchronization in human posterior parietal cortex during reach planning. J Neurosci 30:1402-1412. 